This item was submitted to Loughborough's Research Repository by the author.

Items in Figshare are protected by copyright, with all rights reserved, unless otherwise indicated.

\title{
d-wave superconductivity from electron-phonon interactions
}

PLEASE CITE THE PUBLISHED VERSION

PUBLISHER

(C) American Physical Society

LICENCE

CC BY-NC-ND 4.0

REPOSITORY RECORD

Hague, J.P.. 2019. "D-wave Superconductivity from Electron-phonon Interactions". figshare. https://hdl.handle.net/2134/1352. 


\title{
$d$-wave superconductivity from electron-phonon interactions
}

\author{
J. P. Hague \\ Department of Physics and Astronomy, University of Leicester, Leicester LE1 7RH, United Kingdom \\ and Department of Physics, Loughborough University, Loughborough LE11 3TU, United Kingdom
}

(Received 29 November 2005; published 15 February 2006)

\begin{abstract}
I examine electron-phonon mediated superconductivity in the intermediate coupling and phonon frequency regime of the quasi-two-dimensional Holstein model. I use an extended Migdal-Eliashberg theory that includes vertex corrections and spatial fluctuations. I find a $d$-wave superconducting state that is unique close to half filling. The order parameter undergoes a transition to $s$-wave superconductivity on increasing filling. I explain how the inclusion of both vertex corrections and spatial fluctuations is essential for the prediction of a $d$-wave order parameter. I then discuss the effects of a large Coulomb pseudopotential on the superconductivity (such as is found in contemporary superconducting materials like the cuprates), which results in the destruction of the $s$-wave states, while leaving the $d$-wave states unmodified.
\end{abstract}

DOI: 10.1103/PhysRevB.73.060503

PACS number(s): 74.20.-z, 71.10.-w, 71.38.-k

The discovery of high transition temperatures and a $d$-wave order parameter in the cuprate superconductors are remarkable results and have serious implications for the theory of superconductivity. The presence of large Coulomb interactions in the cuprates that have the potential to destroy conventional $s$-wave BCS states has prompted the search for new mechanisms that can give rise to superconductivity. However, electron-phonon mediated superconductivity is still not well understood, especially in lower dimensional systems. In particular, the electron-phonon problem is particularly difficult at intermediate couplings with large phonon frequency (such as found in the cuprates) and the electron-phonon mechanism cannot be fully ruled out. It is therefore of paramount importance to develop new theories to understand electron-phonon mediated superconductivity away from the BCS limit.

The assumption that electron-phonon interactions cannot lead to high transition temperatures and unusual order parameters was made on the basis of calculations from BCS theory, which is a very weak coupling mean-field theory (although, of course, highly successful for pre-1980s superconductors). ${ }^{1}$ In the presence of strong Coulomb interaction, the BCS $s$-wave transition temperature is vastly reduced. However, the recent measurement of large couplings between electrons and the lattice in the cuprate superconductors means that extensions to the conventional theories of superconductivity are required. ${ }^{2-4}$ In particular, low dimensionality, intermediate dimensionless coupling constants of $\sim 1$, and large and active phonon frequencies of $\sim 75 \mathrm{meV}$ mean that BCS or the more advanced Migdal-Eliashberg (ME) theory cannot be applied. In fact, the large coupling constant and a propensity for strong renormalization in twodimensional (2D) systems, indicate that the bare unrenormalized phonon frequency could be several times greater than the measured $75 \mathrm{meV}^{5}$

Here I apply the dynamical cluster approximation (DCA) to introduce a fully self-consistent momentum-dependent self-energy to the electron-phonon problem. ${ }^{5-8}$ Short-ranged spatial fluctuations and lowest-order vertex corrections are included, allowing the sequence of phonon absorption and emission to be reordered once. In particular, the theory used here is second order in the effective electron-electron coupling $U=-g^{2} / M \omega_{0}^{2}$, which provides the correct weak coupling limit from small to large phonon frequencies. ${ }^{14,18}$ In this paper, I include symmetry broken states via the anomalous self-energy to investigate unconventional order parameters such as the $d$ wave. No assumptions are made in advance about the form of the order parameter.

DCA (Refs. 6, 8, and 9) is an extension to the dynamical mean-field theory for the study of low-dimensional systems. To apply the DCA, the Brillouin zone is divided into $N_{C}$ subzones within which the self-energy is assumed to be momentum independent, and cluster Green functions are determined by averaging over the momentum states in each subzone. This leads to spatial fluctuations with characteristic range, $N_{c}^{1 / D}$. In this paper, $N_{c}=4$ is used throughout. This puts an upper bound on the strength of the superconductivity, which is expected to be reduced in larger cluster sizes. ${ }^{10}$ To examine superconducting states, DCA is extended within the Nambu formalism. ${ }^{7,8}$ Green functions and self-energies are described by $2 \times 2$ matrices, with off-diagonal terms relating to the superconducting states. The self-consistent condition is

$$
\begin{gathered}
G\left(\mathbf{K}, i \omega_{n}\right)=\int_{-\infty}^{\infty} d \epsilon \frac{\mathcal{D}_{i}(\epsilon)\left[\zeta\left(\mathbf{K}_{i}, i \omega_{n}\right)-\epsilon\right]}{\left|\zeta\left(\mathbf{K}_{i}, i \omega_{n}\right)-\epsilon\right|^{2}+\phi\left(\mathbf{K}_{i}, i \omega_{n}\right)^{2}}, \\
F\left(\mathbf{K}, i \omega_{n}\right)=-\int_{-\infty}^{\infty} d \epsilon \frac{\mathcal{D}_{i}(\epsilon) \phi\left(\mathbf{K}_{i}, i \omega_{n}\right)}{\left|\zeta\left(\mathbf{K}_{i}, i \omega_{n}\right)-\epsilon\right|^{2}+\phi\left(\mathbf{K}_{i}, i \omega_{n}\right)^{2}},
\end{gathered}
$$

where $\zeta\left(\mathbf{K}_{i}, i \omega_{n}\right)=i \omega_{n}+\mu-\Sigma\left(\mathbf{K}_{i}, i \omega_{n}\right), \mu$ is the chemical potential, $\omega_{n}$ are the Fermionic Matsubara frequencies, $\phi(\mathbf{K}, i \omega)$ is the anomalous self-energy, and $\Sigma(\mathbf{K}, i \omega)$ is the normal self-energy. $G\left(\mathbf{K}, i \omega_{n}\right)$ must obey the lattice symmetry. In contrast, it is only $\left|F\left(\mathbf{K}, i \omega_{n}\right)\right|$ that is constrained by this condition, since $\phi$ is squared in the denominator of Eq. (1). Therefore the sign of $\phi$ can change. For instance, if the anomalous self-energy has the rotational symmetry $\phi(\pi, 0)$ $=-\phi(0, \pi)$, the on-diagonal Green function, which represents the electron propagation, retains the correct lattice symmetry 
(a)

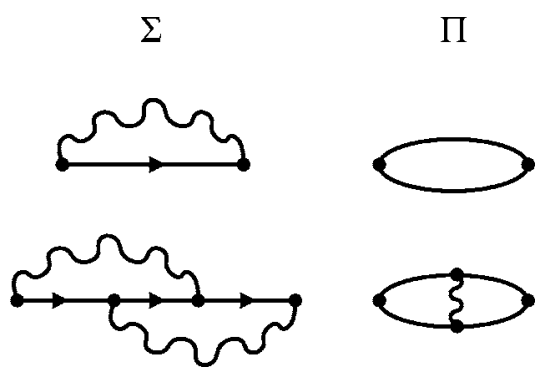

FIG. 1. Diagrammatic representation of the current approximation. Series (a) represents the vertex-neglected theory that corresponds to the Migdal-Eliashberg approach, valid when the phonon energy $\omega_{0}$ and electron-phonon coupling $U$ are small compared to the Fermi energy. Series (b) represents additional diagrams for the vertex corrected theory. The phonon self-energies are labeled with $\Pi$, and $\Sigma$ denotes the electron self-energies. Lines represent the full electron Green function and wavy lines the full phonon Green function.

$G(\pi, 0)=G(0, \pi)$. Therefore, only inversion symmetry is required of the anomalous Green function representing superconducting pairs and the anomalous self-energy.

Here I examine the Holstein model ${ }^{11}$ of electron-phonon interactions. It treats phonons as nuclei vibrating in a timeaveraged harmonic potential (representing the interactions between all nuclei), i.e., only one frequency $\omega_{0}$ is considered. The phonons couple to the local electron density via a momentum-independent coupling constant $g .{ }^{11}$

$$
H=-\sum_{\langle i j\rangle \sigma} t c_{i \sigma}^{\dagger} c_{j \sigma}+\sum_{i \sigma} n_{i \sigma}\left(g r_{i}-\mu\right)+\sum_{i}\left(\frac{M \omega_{0}^{2} r_{i}^{2}}{2}+\frac{p_{i}^{2}}{2 M}\right) \text {. }
$$

The first term in this Hamiltonian represents hopping of electrons between neighboring sites and has a dispersion $\epsilon_{\mathbf{k}}$ $=-2 t \sum_{i=1}^{D} \cos \left(k_{i}\right)$. The second term couples the local ion displacement, $r_{i}$ to the local electron density. The last term is the bare phonon Hamiltonian, i.e., a simple harmonic oscillator. The creation and annihilation of electrons is represented by $c_{i}^{\dagger}\left(c_{i}\right), p_{i}$ is the ion momentum, and $M$ the ion mass. The effective electron-electron interaction is

$$
U\left(i \omega_{s}\right)=\frac{U \omega_{0}^{2}}{\omega_{s}^{2}+\omega_{0}^{2}},
$$

where $\omega_{s}=2 \pi s T, s$ is an integer, and $U=-g^{2} / M \omega_{0}^{2}$ represents the magnitude of the effective electron-electron coupling. $D=2$ with $t=0.25$, resulting in a noninteracting bandwidth $W=2$. A small interplanar hopping $t_{\perp}=0.01$ is included. This is necessary to stabilize superconductivity, which is not permitted in a pure $2 \mathrm{D}$ system. $^{12}$

Perturbation theory in the effective electron-electron interaction (Fig. 1) is applied to second order in $U$, using a skeleton expansion. The electron self-energy has two terms, $\Sigma_{\mathrm{ME}}(\omega, \mathbf{K})$ neglects vertex corrections [Fig. 1(a)], and $\Sigma_{\mathrm{VC}}(\omega, \mathbf{K})$ corresponds to the vertex corrected case [Fig. $1(\mathrm{~b})] . \Pi_{\mathrm{ME}}(\omega, \mathbf{K})$ and $\Pi_{\mathrm{VC}}(\omega, \mathbf{K})$ correspond to the equivalent phonon self-energies. At large phonon frequencies, all second-order diagrams including $\Sigma_{\mathrm{VC}}$ are essential for the

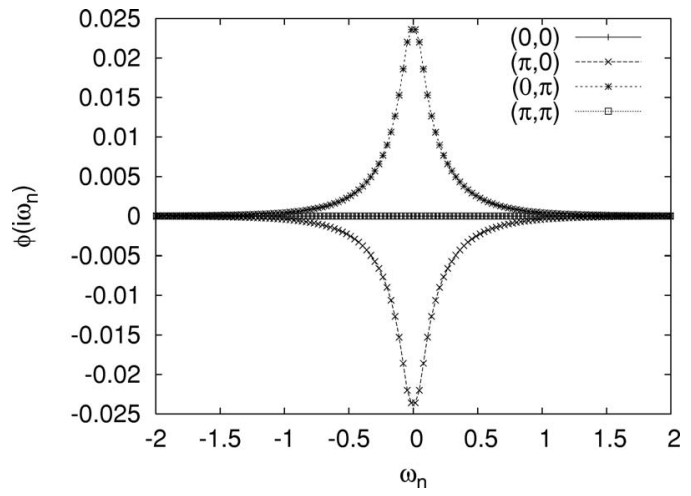

FIG. 2. Anomalous self-energy at half filling. The anomalous self-energy is real. It is clear that $\phi(\pi, 0)=-\phi(0, \pi)$. This is characteristic of $d$-wave order. Similarly, the electron self-energy has the correct lattice symmetry $\Sigma(\pi, 0)=\Sigma(0, \pi)$, which was not imposed from the outset. The gap function is related to the anomalous selfenergy via $\phi\left(i \omega_{n}\right) / Z\left(i \omega_{n}\right)$.

correct description of the weak coupling limit.

The phonon propagator $D(z, \mathbf{K})$ is calculated from

$$
D\left(i \omega_{s}, \mathbf{K}\right)=\frac{\omega_{0}^{2}}{\omega_{s}^{2}+\omega_{0}^{2}-\Pi\left(i \omega_{s}, \mathbf{K}\right)}
$$

and the Green function from Eqs. (1) and (2). $\underset{\Sigma}{\Sigma}=\sum_{\mathrm{ME}}$ $+\Sigma_{\mathrm{VC}}$ and $\Pi=\Pi_{\mathrm{ME}}+\Pi_{\mathrm{VC}}$. Details of the translation of the diagrams in Fig. 1 and the iteration procedure can be found in Ref. 7. Calculations are carried out along the Matsubara axis, with sufficient Matsubara points for an accurate calculation. The equations were iterated until the normal and anomalous self-energies converged to an accuracy of approximately 1 part in $10^{3}$.

Since the anomalous Green function is proportional to the anomalous self-energy, initializing the problem with the noninteracting Green function leads to a nonsuperconducting (normal) state. A constant superconducting field with $d$-wave symmetry was applied to the system to induce superconductivity. The external field was then completely removed. Iteration continued without the field until convergence. This solution was then used to initialize self-consistency for other similar values of the parameters. The symmetry conditions used in Refs. 5 and 7 have been relaxed to reflect the additional breaking of the anomalous lattice symmetry in the $d$-wave state. This does not affect the normal-state Green function, but does affect the anomalous-state Green function.

In Fig. 2, the anomalous self-energy is examined for $n$ $=1.0$ (half filling). The striking feature is that stable $d$-wave superconductivity is found. This is manifested through a change in sign of the anomalous self-energy, which is negative at the $(\pi, 0)$ point and positive at the $(0, \pi)$ point. The electron Green function [Eq. (1)] depends on $\phi^{2}$, so causality and lattice symmetry are maintained. Since the gap function $\phi\left(i \omega_{n}\right) / Z\left(i \omega_{n}\right)$ is directly proportional to $\phi\left(i \omega_{n}\right)$, and $Z\left(i \omega_{n}, \mathbf{K}_{(\pi, 0)}\right)=Z\left(i \omega_{n}, \mathbf{K}_{(0, \pi)}\right)$, then the sign of the order parameter, i.e., the sign of the superconducting gap changes under $90^{\circ}$ rotation. $Z\left(i \omega_{n}\right)=1-\Sigma\left(i \omega_{n}\right) / i \omega_{n}$. 


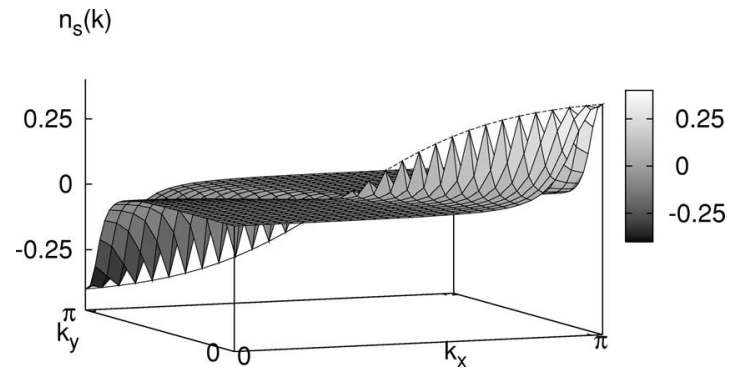

FIG. 3. Variation of superconducting (anomalous) pairing density across the Brillouin zone. $n_{s}(\mathbf{k})=T \Sigma_{n} F\left(i \omega_{n}, \mathbf{k}\right) . U=0.6, \omega_{0}$ $=0.4, n=1$, and $T=0.005$. The $d$-wave order can be seen very clearly, with a change in sign on $90^{\circ}$ rotation and a node situated at the $(\pi / 2, \pi / 2)$ point. The largest anomalous (superconducting) densities are at the $(\pi, 0)$ and $(0, \pi)$ points.

Figure 3 shows the variation of superconducting pairing across the Brillouin zone. $n_{s}(\mathbf{k})=T \Sigma_{n} F\left(i \omega_{n}, \mathbf{k}\right) . U=0.6, \omega_{0}$ $=0.4, n=1$, and $T=0.005$. The $d$-wave order can be seen very clearly. The largest anomalous densities are at the $(\pi, 0)$ and $(0, \pi)$ points, with a node situated at the $(\pi / 2, \pi / 2)$ point and a sign change on $90^{\circ}$ rotation. Pairing clearly occurs between electrons close to the Fermi surface.

So far, the model has been analyzed at half filling. Figure 4 demonstrates the evolution of the order parameter as the number of holes is first increased, and then decreased. The total magnitude of the anomalous density, $n_{s}=\Sigma_{\mathbf{K}}\left|n_{s}\left(\mathbf{K}_{i}\right)\right|$ is examined. When the number of holes is increased, stable $d$-wave order persists to a filling of $n=1.18$, while decreasing monotonically. At the critical point, there is a spontaneous transition to $s$-wave order. Starting from a high filling, and reducing the number of holes, there is a spontaneous transition from $s$-wave to $d$-wave order at $n=1.04$. There is, therefore, hysteresis associated with the self-consistent solution. It is reassuring that the $d$-wave state can be induced without the need for the external field. As previously established, $s$-wave order does not exist at half filling as a manifestation of Hohenberg's theorem, ${ }^{7}$ so the computed $d$-wave order at half filling is the ground state of the model. It is interesting that the $d$ and $s$ channels are able to coexist, considering that the BCS channels are separate on a square lattice. This is due to the vertex corrections, since the self-consistent equations are no longer linear in the gap function (the first-order gap equation vanishes in the $d$-wave case, leaving second-order terms as the leading contribution).

I finish with a brief discussion of Coulomb effects. In the Eliashberg equations, a Coulomb pseudopotential may be added to the theory as

$$
\phi_{C}=U_{C} T \sum_{\mathbf{K}, n} F\left(i \omega_{n}, \mathbf{K}\right) .
$$

It is easy to see the effect of $d$-wave order on this term. Since the sign of the anomalous Green function is modulated, the average effect of $d$-wave order is to nullify the Coulomb contribution to the anomalous self-energy (i.e., $\phi_{C d}=0$ ). This demonstrates that the $d$-wave state is stable to Coulomb perturbations, presumably because the pairs are distance separated. In contrast, the $s$-wave state is not stable to Coulomb

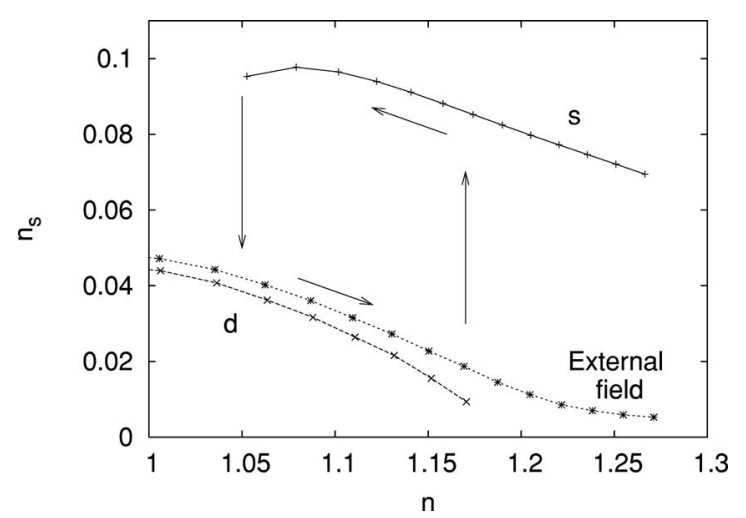

FIG. 4. Hysteresis of the superconducting order parameters. $n_{s}$ $=\Sigma_{\mathbf{K}}\left|n_{s}\left(\mathbf{K}_{i}\right)\right|$. Starting from a $d$-wave state at half filling, increasing the chemical potential increases the filling and decreases the $d$-wave order. Eventually, at $n=1.18$ the system changes to an $s$-wave state. On return from large filling, the $s$-wave superconductivity is persistent to a low filling of $n=1.04$, before spontaneously reverting to a $d$-wave state. The system is highly susceptible to $d$-wave order, and application of a very small external superconducting field to an $s$-wave state results in a $d$-wave state. Note that $d$-wave and $s$-wave channels are coupled in the higher-order theory, so the transition can take place spontaneously, unlike in the standard gap equations.

interaction, with a corresponding reduction of the transition temperature $\left(T_{C}=0\right.$ for $\left.\lambda<\mu_{C}\right)$. Thus, such a Coulomb filter selects the $d$-wave state (see, e.g., Ref. 13). Since large local Coulomb repulsions are present in the cuprates (and indeed most transition metal oxides), then this mechanism seems the most likely to remove the hysteresis. Without the Coulomb interactions, it is expected that the $s$-wave state will dominate for $n>1.04$, since the anomalous order is larger.

I note that a further consequence of strong Coulomb repulsion is antiferromagnetism close to half filling. Typically magnetic fluctuations act to suppress phonon mediated superconducting order. As such, one might expect a suppression of superconducting order close to half filling, with a maximum away from half filling. The current theory could be extended to include additional anomalous Green functions related to antiferromagnetic order. This would lead to a 4 $\times 4$ Green function matrix. A full analysis of antiferromagnetism and the free energy will be carried out at a later date.

In summary, I have carried out simulations of the 2D Holstein model in the superconducting state. Vertex corrections and spatial fluctuations were included in the approximation for the self-energy. The anomalous self-energy and superconducting order parameter were calculated. Remarkably, stable superconducting states with $d$-wave order were found at half filling. $d$-wave states persist to $n=1.18$, where the symmetry of the parameter changes to $s$ wave. Starting in the $s$-wave phase and reducing the filling, $d$-wave states spontaneously appear at $n=1.04$. The spontaneous appearance of $d$-wave states in a model of electron-phonon interactions is of particular interest, since it may negate the need for unconventional pairing mechanisms in the cuprates. ${ }^{17,19}$

The inclusion of vertex corrections and spatial fluctuations was essential to the emergence of the $d$-wave states in the Holstein model, which indicates why BCS and ME calculations do not predict this phenomenon. For very weak 
coupling, the off-diagonal Eliashberg self-energy has the form $-U T \Sigma_{\mathbf{Q}, n} F\left(i \omega_{n}, \mathbf{Q}\right) D_{0}\left(i \omega_{s}-i \omega_{n}\right)$, so it is clear (for the same reasons as the Coulomb pseudopotential) that this diagram has no contribution in the $d$-wave phase (the weak coupling phonon propagator is momentum independent for the Holstein model). Therefore, vertex corrections are the leading term in the weak coupling limit. Furthermore, I have discussed the inclusion of Coulomb states to lowest order, which act to destabilize the $s$-wave states, while leaving the $d$-wave states unchanged. Since the Coulomb pseudopotential has no effect, then it is possible that electron-phonon interactions are the mechanism inducing $d$-wave states in real materials such as the cuprates. The Coulomb filtering mechanism works for $p$-wave symmetry and higher, so it is possible that electron-phonon interactions could explain many superconductors. Certainly, such a mechanism cannot be ruled out. The doping dependence of the order qualitatively matches that of $\mathrm{La}_{2-x} \mathrm{Sr}_{x} \mathrm{CuO}_{4}$ (here order extends to $x=0.18$, in the Cuprate to $x=0.3$ ). Antiferromagnetism is only present in the cuprate very close to half filling (up to approx $x=0.02$ ), and on a mean-field level does not interfere with the $d$-wave superconductivity at larger dopings.

It has been determined experimentally that strong electron-phonon interactions and high phonon frequencies are clearly visible in the electron and phonon band structures of the cuprates, and are, therefore, an essential part of the physics. ${ }^{3,4}$ Similar effects to those observed in the cuprates are seen in the electron and phonon band structures of the 2D Holstein model in the normal phase. ${ }^{5}$ It is clearly of interest to determine whether other features and effects in the cuprate superconductors could be explained with electron-phonon interactions alone.

I thank the University of Leicester for hospitality while carrying out this work. I thank E. M. L. Chung for useful discussions. This work was partially supported under EPSRC Grant No. EP/C518365/1.
${ }^{1}$ J. Bardeen, L. N. Cooper, and J. R. Schrieffer, Phys. Rev. 108, 1175 (1957).

${ }^{2}$ G. M. Zhao, M. B. Hunt, H. Keller, and K. A. Müller, Nature (London) 385, 236 (1997).

${ }^{3}$ A. Lanzara et al., Nature (London) 412, 6846 (2001).

${ }^{4}$ R. J. McQueeney, Y. Petrov, T. Egami, M. Yethiraj, G. Shirane, and Y. Endoh, Phys. Rev. Lett. 82, 628 (1999).

${ }^{5}$ J. P. Hague, J. Phys.: Condens. Matter 15, 2535 (2003).

${ }^{6}$ M. H. Hettler, A. N. Tahvildar-Zadeh, M. Jarrell, T. Pruschke, and H. R. Krishnamurthy, Phys. Rev. B 58, R7475 (1998).

${ }^{7}$ J. P. Hague, J. Phys.: Condens. Matter 17, 5663 (2005).

${ }^{8}$ T. Maier, M. Jarrell, T. Pruschke, and M. H. Hettler, Rev. Mod. Phys. 77, 1027 (2005).

${ }^{9}$ M. H. Hettler, M. Mukherjee, M. Jarrell, and H. R. Krishnamurthy, Phys. Rev. B 61, 12739 (2000).

${ }^{10}$ M. Jarrell, Th. Maier, C. Huscroft, and S. Moukouri, Phys. Rev. B 64, 195130 (2001).

${ }^{11}$ T. Holstein, Ann. Phys. 8, 325 (1959).
${ }^{12}$ P. C. Hohenberg, Phys. Rev. 158, 383 (1967).

${ }^{13}$ J. F. Annett, Superconductivity, Superfluidity and Condensates (Oxford University Press, Oxford, 2004).

${ }^{14}$ C. Grimaldi, L. Pietronero, and S. Strässler, Phys. Rev. Lett. 75, 1158 (1995).

${ }^{15}$ A. A. Abrikosov, Physica C 244, 243 (1995).

${ }^{16}$ A. A. Abrikosov, Phys. Rev. B 52, R15738 (1995).

${ }^{17}$ R. J. Birgeneau and G. Shirane, in Physical Properties of High Temperature Superconductors I, edited by D. M. Ginsberg (World Scientific, Singapore, 1989).

${ }^{18}$ I also note the extensions to Eliashberg theory carried out by Grimaldi et al. (Ref. 14).

${ }^{19} \mathrm{On}$ the basis of a screened electron-phonon interaction, Abrikosov claims to have found stable $d$-wave states in a BCS-like theory (Refs. 15 and 16). However, with an unscreened Holstein potential, the transition temperature, the $d$-wave channel given by the standard theory is zero. Also, it is not completely clear that the assumed order parameter in his work has $d$-wave symmetry. 